\title{
Perception de la fréquence d'apparition des comportements perturbateurs par des stagiaires en enseignement de l'éducation physique et à la santé (EPS)
}

\author{
Jean-François Desbiens, Sèverine Lanoue, Sylvain Turcotte, Jean-Sébastien \\ Tourigny et Carlo Spallanzani
}

Volume 12, numéro 2, 2009

URI : https://id.erudit.org/iderudit/1017466ar

DOI : https://doi.org/10.7202/1017466ar

\section{Aller au sommaire du numéro}

\section{Éditeur(s)}

Faculté d'éducation, Université de Sherbrooke

\section{ISSN}

1911-8805 (numérique)

\section{Découvrir la revue}

\section{Citer cet article}

Desbiens, J.-F., Lanoue, S., Turcotte, S., Tourigny, J.-S. \& Spallanzani, C. (2009). Perception de la fréquence d'apparition des comportements perturbateurs par des stagiaires en enseignement de l'éducation physique et à la santé (EPS). Nouveaux cahiers de la recherche en éducation, 12(2), 179-193. https://doi.org/10.7202/1017466ar

\section{Résumé de l'article}

Les enseignants débutants en éducation physique et à la santé (EPS) ont des habiletés insuffisantes pour gérer efficacement la complexité des situations d'apprentissage. Leurs capacités d'observer les éléments pertinents des situations, de les traiter et de les interpréter sont limitées. Cette étude consacrée à des stagiaires finissants avait pour objectifs spécifiques de : 1 ) comparer la fréquence perçue et effective des comportements perturbateurs (CP) selon leur nature; 2) comparer la fréquence perçue et effective des CP selon leur moment d'apparition. Les résultats montrent que les perceptions des stagiaires finissants sont variables et qu'elles ne correspondent pas fidèlement aux CP effectivement dénombrés selon le degré d'intensité de ces derniers. Cependant, elles reflètent de manière fiable leur moment d'apparition. Le poids des CP dans l'économie de la séance n'est apparemment pas uniquement lié à leur seule fréquence, mais à d'autres facteurs qui restent à approfondir. Les auteurs proposent en conclusion des pistes d'améliorations des dispositifs de formation initiale à l'enseignement afin que les stagiaires deviennent de meilleurs observateurs et interprètes des comportements des élèves.
Tous droits réservés (C Faculté d'éducation, Université de Sherbrooke,

Ce document est protégé par la loi sur le droit d'auteur. L'utilisation des services d'Érudit (y compris la reproduction) est assujettie à sa politique d'utilisation que vous pouvez consulter en ligne.

https://apropos.erudit.org/fr/usagers/politique-dutilisation/ 


\title{
Perception de la fréquence d'apparition des comportements perturbateurs par des stagiaires en enseignement de l'éducation physique et à la santé (EPS)
}

\author{
Jean-François Desbiens, Sèverine Lanoue, Sylvain Turcotte, \\ Jean-Sébastien Tourigny et Carlo Spallanzani
}

Université de Sherbrooke

Résumé - Les enseignants débutants en éducation physique et à la santé (EPS) ont des habiletés insuffisantes pour gérer efficacement la complexité des situations d'apprentissage. Leurs capacités d'observer les éléments pertinents des situations, de les traiter et de les interpréter sont limitées. Cette étude consacrée à des stagiaires finissants avait pour objectifs spécifiques de:1) comparer la fréquence perçue et effective des comportements perturbateurs (CP) selon leur nature; 2) comparer la fréquence perçue et effective des $\mathrm{CP}$ selon leur moment d'apparition. Les résultats montrent que les perceptions des stagiaires finissants sont variables et qu'elles ne correspondent pas fidèlement aux CP effectivement dénombrés selon le degré d'intensité de ces derniers. Cependant, elles reflètent de manière fiable leur moment d'apparition. Le poids des CP dans l'économie de la séance n'est apparemment pas uniquement lié à leur seule fréquence, mais à d'autres facteurs qui restent à approfondir. Les auteurs proposent en conclusion des pistes d'améliorations des dispositifs de formation initiale à l'enseignement afin que les stagiaires deviennent de meilleurs observateurs et interprètes des comportements des élèves.

Abstract - Novice physical-education teachers have insufficient skill to effectively manage the complexity of learning situations. Their ability to observe a situation's relevant components, process them, and interpret them is limited. The objectives of this study of student-teachers in their graduating year were to: 1) compare the perceived and actual frequencies of pupils' disruptive behaviours, by kind; 2) compare the perceived and actual frequencies of disruptive behaviours, by moment of occurrence. The results show that the perceptions of student-teachers in their final year vary and that they do not closely correspond to the actual count of disruptive behaviours based on level of intensity. On the other hand, they do faithfully reflect the behaviours' moments of occurrence. The prominence of the role played by disruptive behaviours in class management would appear to be linked not just to their frequency of occurrence but also to other factors, which remain to be examined. In concluding, the authors suggest approaches to improving pre-service education conditions so that student-teachers can become better observers and interpreters of pupils' behaviours. 


\section{Introduction}

Dans un article publié il y a déjà plus de vingt ans, Doyle (1986) a décrit la classe comme un environnement complexe caractérisé par la multidimensionnalité, la simultanéité, l'immédiateté, l'imprévisibilité, l'accessibilité ainsi que l'historicité des évènements qui y surviennent. Composer avec l'ensemble de ces caractéristiques exige donc des enseignants qu'ils soient capables d'utiliser avec efficacité et à propos plusieurs habiletés essentielles en matière de gestion de classe comme la vigilance et la répartition de l'attention. Ces deux habiletés cognitives sont à la base d'une supervision active efficace (Desbiens, 2002; Desbiens et Spallanzani, 2005) et leur importance au regard du maintien de l'implication des élèves dans les tâches prescrites a déjà été démontrée par Copeland (1987). Or, il appert que les enseignants stagiaires ainsi que les débutants en enseignement de l'éducation physique ont bien souvent des habiletés insuffisantes pour gérer efficacement toute la complexité que recèlent les situations éducatives dans cette discipline (Fernandez-Balboa, 1991).

Ces «insuffisances professionnelles» (Martineau, Gauthier et Desbiens, 2000, p. 316) entrainent parfois des pertes de contrôle du fonctionnement du groupe d'élèves, lesquelles provoquent en retour des pertes de temps. L'indiscipline des élèves et leur démotivation figurent d'ailleurs parmi les plus importants problèmes avec lesquels les enseignants débutants doivent composer (Legault, 1999). Il n'est donc pas étonnant d'apprendre que ceux-ci soient vécus surtout négativement par les stagiaires (Fortier et Desrosiers, 1991) et que la gestion des comportements non appropriés des élèves soit pour eux l'une des principales sources d'anxiété (Goyette, Doré et Dion, 1997; Siedentop, 1994).

Pour Chouinard (1999) et Kokkinos, Panayiotou et Davazoglou (2004), ces difficultés seraient explicables par le fait que les stagiaires et les enseignants débutants ont des représentations inopérantes à propos des élèves, des rapports à entretenir avec eux, du processus d'enseignement-apprentissage, des contenus et de l'organisation de la classe. De plus, leurs automatismes cognitifs et leurs connaissances au plan procédural leur permettant d'établir et de maintenir l'ordre, de résoudre des problèmes, d'engager et de maintenir les élèves impliqués dans les tâches sont souvent insuffisants.

Cette insistance sur les dimensions cognitives qui règlent la qualité de l'intervention éducative est retrouvée dans plusieurs recherches publiées au cours des vingt dernières années. FernandezBalboa (1991), par exemple, insiste sur le fait que les croyances ou représentations à propos de l'enseignement, les pensées et les décisions prises en cours d'interaction et les pensées à visées de planification ou d'anticipation influencent l'action des enseignants et que, dans le cas particulier des inconduites des élèves

les processus mentaux sont des facteurs cruciaux affectant leurs actions parce qu'un comportement perturbateur n'est pas une propriété d'une action, mais plutôt une action en contexte [...] il est raisonnable de penser que les processus mentaux des enseignants novices concernant les comportements perturbateurs peuvent être d'importants facteurs affectant l'efficacité de leurs interventions disciplinaires. (Fernandez-Balboa, 1991, p. 60) (traduction libre)

Certains (Kokkinos et al., 2004) suggèrent même que l'anxiété ressentie par les stagiaires face à l'indiscipline des élèves serait davantage fonction de la manière dont ils perçoivent et interprètent les comportements de ces derniers qu'une représentation exacte de leur sévérité et des problèmes qu'ils posent au plan de leur gestion. 
De son côté, Copeland (1987) conclut que la tendance des stagiaires en enseignement à gérer leur classe de manière à réduire au minimum les comportements hors tâche des élèves résulterait de l'interaction entre leurs habiletés à traiter adéquatement les innombrables informations présentes dans le système-classe, leur compréhension de l'histoire de ce collectif et du degré de sophistication des structures conceptuelles utilisées pour donner sens à cet écosystème dynamique (Desbiens et Spallanzani, 2005).

Cela suppose à la base que les stagiaires sachent quoi regarder ou quoi rechercher. Des travaux publiés par Bell, Barrett et Allison (1985) ont montré à cet effet que, malgré une posture d'extériorité (observateurs non participants) par rapport aux situations d'enseignement-apprentissage, les stagiaires en enseignement de l'éducation physique demandent d'être guidés dans leurs observations faute de quoi il y a de fortes chances qu'ils observent un spectre trop restreint d'événements ou qu'ils n'échappent carrément des éléments jugés importants au regard de l'intervention en classe. Dans la même veine, Turcotte et Spallanzani (2007) ont constaté qu'une divergence importante existe entre l'autoanalyse des situations professionnelles vécues lors du stage et l'analyse réalisée a posteriori en groupe lors d'épisodes dirigés par des personnes-ressources. En contrepartie, Belka (1988) a constaté qu'à mesure que les stagiaires avancent dans leur programme d'études, ils apprennent à observer et à interpréter d'une manière plus complexe les situations qui leur sont présentées.

Malgré ce qui précède, les schémas interprétatifs des enseignants novices demeurent moins élaborés, moins interconnectés et moins facilement accessibles que ceux des enseignants experts (Graham, French et Woods, 1993). Les habiletés des premiers en matière de raisonnement pédagogique et leurs connaissances de contenu pédagogique seraient également moins développées que celles des seconds, voire inexistantes. Clairement, les enseignants débutants n'auraient pas les acquis nécessaires pour interpréter les évènements survenant en classe d'une manière signifiante. Très sensibles à l'ambiance dans laquelle les cours se déroulent (Spallanzani et Tousignant, 1990), ils auraient tendance à focaliser sur les dimensions sociales plutôt que sur celles liées à l'enseignement et à l'apprentissage (Graham et al., 1993). Ils seraient également plus attentifs aux comportements perturbateurs (CP) les plus flagrants et dérangeants, mais n'auraient pas encore développé la sensibilité aux aspects signalant des comportements problématiques plus latents (Kokkinos et al., 2004).

Retenons de ce tour d'horizon que, même rendus à la fin de leur parcours en formation initiale, les enseignants stagiaires ne maitrisent pas toutes les habiletés cognitives ni les techniques de supervision active nécessaires pour extraire, traiter et interpréter les informations pertinentes en relation aux évènements qui surviennent en classe. Cela pose des difficultés notamment au plan de la gestion de l'indiscipline des élèves puisqu'il ressort des éléments présentés plus haut que les interventions que les stagiaires décident ou non de mettre en œuvre sont fortement déterminées par leurs manières de comprendre l'écologie de la classe à un moment et par rapport à un contexte donnés.

Comment des enseignants stagiaires finissants du secondaire en éducation physique et à la santé perçoivent-ils cette écologie considérée sous l'angle du degré d'indiscipline qui prévaut durant les cours qu'ils dirigent? Dans quelle mesure cette perception correspond-elle à ce qui s'y déroule effectivement à différents moments d'un cours? Voilà deux questions qui étaient posées au départ de la présente étude. De façon plus précise, il s'agira de: 1) comparer la fréquence perçue et effective des $\mathrm{CP}$ selon leur nature; 2) comparer la fréquence perçue et effective des $\mathrm{CP}$ selon leur moment d'apparition. 


\section{Méthodologie}

Les participants à cette étude ${ }^{1}$ sont 24 stagiaires ( 7 femmes et 17 hommes) en enseignement de l'éducation physique et à la santé (EEPS). Toutes les participantes et tous les participants ont préalablement été informés des visées de l'étude et des dispositions prises par les chercheurs pour préserver leur anonymat ainsi que la confidentialité des données brutes. Leur participation était libre et volontaire. Aucun n'a reçu de rémunération pour sa participation.

Les stagiaires de quatrième année étaient engagés dans un stage intensif de sept semaines tenu à la huitième et dernière session de leur programme de formation. À la conclusion de ce stage et sur la base de la réussite des autres activités de formation au programme, les stagiaires ont obtenu leur brevet d'enseignement et avaient légalement le droit d'exercer le métier d'enseignant en éducation physique et à la santé dans au Québec (Canada). Ils ont été observés par l'équipe de chercheurs à une seule reprise durant la cinquième, la sixième ou la septième semaine de leur stage terminal. Lors des observations, les stagiaires intervenaient auprès d'élèves de secondaire un à cinq (12 à 17 ans) dans le contexte de situations d'enseignement-apprentissage se déroulant en gymnase ou en palestre.

Les données pertinentes à l'étude ont été collectées de deux façons. Dans un premier temps, les $\mathrm{CP}$ effectifs ont été recueillis à l'aide d'un dispositif technologique composé de deux caméscopes et d'un micro sans fil. L'un des caméscopes était équipé d'un objectif grand angle afin de capter le maximum d'éléments de la situation d'enseignement-apprentissage. L'autre caméscope suivait scrupuleusement le stagiaire dans tous ses déplacements et était relié au micro sans fil que celui-ci devait porter afin de recueillir ses verbalisations en cours d'action. Un total de 24 séances de 75 minutes ont été filmées. La stratégie d'analyse des CP effectifs retenue est l'analyse vidéoscopique en différé. L'analyse en différé consiste à visionner les séances d'enseignement à plusieurs reprises en vue de repérer des incidents disciplinaires. Le prélèvement des données relatives aux CP a été réalisé à l'aide du Système d'observation des incidents disciplinaires (SOID) mis au point par Brunelle, Brunelle, Gagnon, Goyette, Martel, Marzouk et Spallanzani (1993) (annexe 1) et légèrement adapté pour les fins de cette recherche.

Dans un second temps, les CP perçus des étudiants stagiaires ont été recueillis à l'aide d'un bref questionnaire auto-administré (annexe 2). Les stagiaires avaient pour consigne de remplir le questionnaire immédiatement après le cours ciblé. Les répondants étaient familiers avec les catégories d'analyse du questionnaire fondées dans une large mesure sur le SOID, car ce système d'analyse avait été utilisé comme cadre de référence lors d'activités de formation tenues plus tôt durant le programme de formation auquel ils étaient inscrits. De manière plus précise, ils avaient pour tâche de : 1) indiquer en ordre croissant les cinq catégories de moments d'apparition des CP, le rang 1 correspondant à la catégorie de moments présentant le moins de $\mathrm{CP}$ et le rang 5 correspondant à la catégorie de moments présentant le plus de $\mathrm{CP}$; 2) préciser la présence ou l'absence de chacun des CP lors de la séance et 3) indiquer l'ordre d'importance des trois niveaux de CP selon la fréquence d'apparition perçue des $\mathrm{CP}$, le rang 1 correspondant au degré d'intensité des CP ayant été le moins fréquent et le rang 3 correspondant à celui ayant été le plus fréquent.

1 Cette étude a été rendue possible grâce à l'obtention d'une subvention ordinaire de recherche du Conseil de la recherche en sciences humaines du Canada (2003-2006, nº dossier 410-2003-1444). 
Le SOID est un système d'observation à catégories prédéterminées. Il permet d'identifier et de décrire le contenu des incidents disciplinaires dont les CP qui surviennent lors d'une séance d'éducation physique et à la santé. Le SOID est basé sur une stratégie d'observation évènementielle, c'est-à-dire que les incidents disciplinaires sont notés selon leur moment d'apparition au cours d'une séance. Ce système d'observation a démontré sa robustesse. Au moment de son élaboration, 6 codeurs ont eu à confronter leurs perceptions relatives à 10 incidents disciplinaires et à chacune des 70 décisions de codage qui leur étaient rattachées. Le pourcentage de fidélité inter-analyste obtenu à ce moment s'est élevé à $88 \%$.

Le système d'observation propose huit catégories: 1) les comportements perturbateurs des élèves; 2) le degré d'intensité (1, 2 ou 3 ) de la perturbation associée aux comportements adoptés par les élèves ; 3 ) les moments d'apparition des différents comportements perturbateurs ; 4) le seuil d'accessibilité du comportement perturbateur pour le stagiaire; 5) l'impact des comportements perturbateurs adoptés par les élèves sur le déroulement de la séance d'enseignement; 6) les types de réaction des stagiaires ; 7) l'effet des réactions des stagiaires sur le comportement perturbateur des élèves et 8) le nombre d'élèves adoptant les comportements ciblés. Dans cette étude, nous nous attarderons seulement aux quatre premières catégories.

- Les comportements perturbateurs que peuvent adopter les élèves lors des séances d'enseignement en éducation physique et à la santé sont au nombre de 19. Ceux-ci sont regroupés en trois niveaux selon le degré d'intensité du comportement et son influence possible sur le bon déroulement de la séance d'enseignement. Les CP de niveau 1 (CPN1) sont ceux qui ont généralement une faible influence sur la vie de la classe mais qui peuvent néanmoins déranger l'enseignant. Les $\mathrm{CP}$ de niveau 2 (CPN2) sont généralement susceptibles de déranger la classe à court ou moyen terme. Les CP de niveau 3 (CPN3) dérangent effectivement le bon déroulement de la classe au moment où ils surviennent.

- Le SOID comporte dix moments facilitant le repérage des incidents disciplinaires. Comme le montre l'annexe 2, ces moments ont été regroupés afin d'en faciliter le traitement. Par exemple, les moments 2 à 5 ont été regroupés sous l'étape de préparation. Les moments 6 et 7 ont été regroupés sous l'étape de réalisation. Les moments 8 et 9 ont été regroupés sous l'étape d'intégration.

- Un CP est considéré accessible s'il se déroule dans le champ visuel du stagiaire ou qu'il est audible par ce dernier. Un CP est considéré non accessible s'il se déroule à l'extérieur du champ visuel du stagiaire ou qu'il est inaudible par ce dernier.

Une équipe de quatre codeurs a été formée à l'utilisation du SOID en vue du codage des bandes vidéoscopiques. Pour s'entrainer, les codeurs ont analysé en équipes de deux, trois séances d'enseignement afin de vérifier leur capacité à repérer les incidents disciplinaires qui surviennent lors des séances d'enseignement en éducation physique et à la santé. Les deux équipes de codeurs ont obtenu un taux d'accord de l'ordre de $82,1 \%$ lors du repérage des incidents disciplinaires. Par ailleurs, des coefficients de fidélité ont été obtenus en calculant le pourcentage d'accords entre les données décrites par les deux équipes de codeurs en fonction de six catégories de la grille d'observation (types de CP, niveau de perturbation, nombre d'élèves, moments d'apparition, impact des CP sur le déroulement des séances, l'accessibilité des CP). Un taux d'accord supérieur à $90 \%$ a été obtenu pour chacune d'elles. L'entrainement s'est poursuivi par l'analyse de six autres bandes étrangères à celles du présent corpus. Au cours de cette démarche, six tests de fidélité ont été exécutés. Les 
codeurs ont analysé, de façon indépendante, les six séances afin de vérifier leur capacité de repérer les incidents disciplinaires survenant lors des séances. Un taux d'accord de $81,7 \%$ a été obtenu quant au repérage des incidents disciplinaires alors qu'un taux d'accord supérieur à $90 \%$ a été obtenu pour le codage des $\mathrm{CP}$ par rapport à chacune des six catégories précitées.

Une base de données correspondant aux différentes catégories de la grille d'observation a permis la saisie des données dans des fichiers SPSS [Appache Software Foundation (version 13), s.a., 2004] en vue de leur analyse au plan statistique. L'analyse a premièrement consisté en un dénombrement des fréquences absolues et relatives des CP par catégories de la grille du SOID. Deuxièmement, nous avons cherché à comparer les fréquences observées et théoriques de modalités de variables catégorielles (accessibilité et non-accessibilité contre intensité des CP) à l'aide du Khi deux, un test d'hypothèse non paramétrique à large spectre (Stafford et Bodson, 2006). Troisièmement, nous avons voulu mesurer la corrélation entre des variables ordinales (perception de la fréquence des CP et fréquences effectives des CP selon leur intensité et selon leur moment d'apparition) par le calcul du coefficient de Spearman (Siegel et Castellan, 1988). Quatrièmement, nous avons voulu vérifier la force des relations entre des variables dichotomiques (perception de la présence ou de l'absence des $19 \mathrm{CP}$ ) et des variables quantitatives (fréquences effectives des 19 CP) par le calcul du coefficient de corrélation point bisériale (Broning et Kintz, 1987; Sprinthall, 2000). Dans tous les cas, un résultat était jugé significatif au plan statistique si la valeur de alpha était inférieure ou égale à 0,05 .

\section{Résultats}

La présentation des résultats s'effectuera en trois temps. Il sera d'abord question d'établir l'accessibilité des CP selon leur degré d'intensité. Ensuite, partant des perceptions des stagiaires et de l'observation en différé à l'aide du SOID, nous examinerons les différences de classement des CP selon leur degré d'intensité et leur moment d'apparition durant la séance.

\subsection{L'accessibilité des comportements perturbateurs}

Les résultats obtenus montrent que $76 \%(\mathrm{~N}=1012 / 1332)$ des CP répertoriés sont accessibles aux stagiaires alors que $24 \%(\mathrm{~N}=320 / 1332)$ ne le sont pas. Lorsque l'on analyse la distribution des CP accessibles et non accessibles en fonction du degré d'intensité des CP (CPN1, CPN2 ou CPN3) à l'aide du Khi deux, il apparait que celle-ci n'est pas liée au hasard et que des différences significatives entre les modalités des catégories sont constatées $\left(X^{2}=31,97 ; d l=2 ; p=0,000\right)$. Les CPN1 sont clairement les plus accessibles. On peut faire l'hypothèse qu'il en est ainsi parce que ces derniers surviennent majoritairement à des moments où l'environnement d'apprentissage est plus stable (explications : 52,5\%; introduction du cours : 18,0\%; conclusion du cours : 5,9\%) alors que les CPN3 sont manifestés davantage à des moments du cours marqués par de l'instabilité (éducatifs : $30,7 \%$; transitions : $28,1 \%$; jeux : $23,7 \%$ ).

\subsection{Différences de classement des CP selon leur degré d'intensité}

Lorsqu'interrogés par questionnaire, immédiatement après leur cours d'éducation physique et à la santé, les stagiaires ( $N=20 ; 4$ sujets exclus en raison de réponses non admissibles au questionnaire) rapportent avoir perçu que les CPN1 sont les plus fréquents suivis des CPN3 et des CPN2 (tableau 1). 
Tableau 1

Classement en rang des comportements perturbateurs perçus obtenus à l'aide d'un questionnaire et effectifs obtenus à l'aide du SOID selon le degré d'intensité des comportements perturbateurs

\begin{tabular}{|c|c|c|c|c|c|c|}
\hline \multirow[t]{2}{*}{ Intensité des CP } & \multirow[t]{2}{*}{$\begin{array}{l}\text { Rang } \\
\text { attribué }\end{array}$} & \multicolumn{2}{|c|}{$\begin{array}{c}\text { Fréquence d'attribution } \\
\text { des rangs par les stagiaires }\end{array}$} & \multirow[t]{2}{*}{$\begin{array}{l}\text { Rang } \\
\text { attribué }\end{array}$} & \multicolumn{2}{|c|}{$\begin{array}{l}\text { Fréquences d'apparition } \\
\text { effective des (CP) }\end{array}$} \\
\hline & & Nbre Stag. & $\%$ Stag. & & F. brute & F. $\%$ \\
\hline \multirow[t]{3}{*}{ CPN1 (Faible intensité) } & \multirow[t]{3}{*}{3} & R.1: 5 & 22,73 & \multirow[t]{3}{*}{1} & \multirow[t]{3}{*}{255} & \multirow[t]{3}{*}{19,14} \\
\hline & & R.2: 5 & 22,73 & & & \\
\hline & & R.3: 12 & 54,54 & & & \\
\hline \multirow[t]{3}{*}{ CPN2 (Intensité modérée) } & \multirow[t]{3}{*}{1} & R.1: 5 & 22,73 & \multirow[t]{3}{*}{2} & \multirow[t]{3}{*}{389} & \multirow[t]{3}{*}{29,20} \\
\hline & & R.2: 13 & 59,09 & & & \\
\hline & & R.3: 4 & 18,18 & & & \\
\hline \multirow[t]{3}{*}{ CPN3 (Intensité élevée) } & \multirow[t]{3}{*}{2} & R.1: 12 & 54,54 & \multirow[t]{3}{*}{3} & \multirow[t]{3}{*}{688} & \multirow[t]{3}{*}{51,66} \\
\hline & & R.2: 4 & 18,18 & & & \\
\hline & & R.3: 6 & 27,26 & & & \\
\hline Total & ---- & ----- & ---- & $\begin{array}{ll}---- \\
---\end{array}$ & 1332 & 100 \\
\hline
\end{tabular}

Note 1. Rang 1: moins fréquent; rang 3 : plus fréquent.

Note 2. CPNx: comportement perturbateur de degré 1, 2 ou 3 selon le cas.

Cet ordre établi selon les réponses des stagiaires en fonction de la fréquence d'apparition perçue des CP (1: moins fréquent; 3 : plus fréquent) est presque totalement à l'inverse de celui établi par le dénombrement en différé des CP à l'aide du SOID. Selon ce dernier, les CPN3 ( $\mathrm{N}=688 ; 51,66 \%)$ obtiennent un rang 3 (plus fréquent), les CPN2 ( $\mathrm{N}=353 ; 29,20 \%)$ se voient attribuer un rang 2 et les CPN1 ( $\mathrm{N}=232 ; 19,14 \%)$ obtiennent un rang 1 (moins fréquent). Le calcul de la corrélation de rang de Spearman permet d'établir qu'une relation de $r_{s}=-0,5$ (alpha $>0,05$ ) existe entre les perceptions des stagiaires et la fréquence effective des CP selon leur degré d'intensité. Cette corrélation n'est pas significative en raison du faible nombre d'observations. Le signe négatif de celle-ci indique néanmoins que les deux variables tendent à se comporter de manière opposée et donc que les perceptions des CP par les stagiaires ne correspondent pas à la réalité décrite par le SOID.

Une analyse corrélationnelle plus fine des relations entre les perceptions de la présence ou de l'absence des CP durant le cours par les stagiaires et la fréquence effective d'apparition de chacun des $19 \mathrm{CP}$ a ensuite été réalisée. Il ressort que des corrélations significatives sont trouvées dans 5 cas sur 19. Les coefficients de corrélation point bisérial les plus élevés obtenus concernent le CPN3 «Est grossier» $\left(r_{p . b}=0,62 ; \mathrm{t}=3,73 ; \mathrm{p}<0,05\right)$ et le CPN1 «Quitte la salle» $\left(r_{p . b}=0,51\right.$; $\mathrm{t}=2,76 ; \mathrm{p}<0,05)$ ainsi que dans l'ordre: le CPN3 «S'en prend au matériel» $\left(r_{p . b}=0,42 ; \mathrm{t}=2,17\right.$; $\mathrm{p}<0,05)$ et les CPN1 «Arrive en retard» $\left(r_{p . b}=0,41 ; \mathrm{t}=3,73 ; \mathrm{p}<0,05\right)$ ainsi que «Pas de costume» $\left(r_{p . b}=0,41 ; \mathrm{t}=2,12 ; \mathrm{p}<0,05\right)$. Ces corrélations significatives signifient que, dans ces cas précis, les perceptions des stagiaires semblent représentatives de la réalité décrite par le SOID quant à la fréquence effective des CP. Dans les autres cas, des divergences importantes entre les deux types de variables sont observées laissant entendre que les perceptions ne représentent pas de manière fiable cette même réalité. 


\subsection{Différences de classement des CP selon le moment d'apparition}

Selon les stagiaires, les CP considérés indistinctement de leur degré d'intensité surviennent plus fréquemment (rangs 4 et 5 combinés) durant les étapes de préparation et de réalisation des cours d'éducation physique et à la santé alors que c'est après le cours que surviennent le moins de CP (tableau 2). L'étape d'intégration arrive au troisième rang quant à la fréquence effective des $\mathrm{CP}$ alors que le moment «Avant le cours » se place à l'avant-dernier rang.

Tableau 2

Classement en rang des comportements perturbateurs perçus obtenus à l'aide d'un questionnaire et effectifs obtenus à l'aide du SOID selon le moment du cours

\begin{tabular}{|c|c|c|c|c|c|c|}
\hline \multirow[t]{2}{*}{ Moment du cours } & \multirow[t]{2}{*}{$\begin{array}{l}\text { Rang } \\
\text { attribué }\end{array}$} & \multicolumn{2}{|c|}{$\begin{array}{l}\text { Fréquence d'attribution des } \\
\text { rangs par les stagiaires }\end{array}$} & \multirow[t]{2}{*}{$\begin{array}{l}\text { Rang } \\
\text { attribué }\end{array}$} & \multicolumn{2}{|c|}{$\begin{array}{c}\text { Fréquences d'apparition } \\
\text { effective des (CP) }\end{array}$} \\
\hline & & Nbre Stag. & $\%$ Stag. & & F. brute & F. \% \\
\hline \multirow[t]{5}{*}{ Avant le cours } & \multirow[t]{5}{*}{2} & R.1: 8 & 40 & \multirow[t]{5}{*}{2} & \multirow[t]{5}{*}{22} & \multirow[t]{5}{*}{1,66} \\
\hline & & R.2: 4 & 20 & & & \\
\hline & & R.3: 3 & 15 & & & \\
\hline & & R. $4: 3$ & 15 & & & \\
\hline & & R.5: 2 & 10 & & & \\
\hline \multirow[t]{5}{*}{ Étape de préparation } & \multirow[t]{5}{*}{5} & R.1: 2 & 10 & \multirow[t]{5}{*}{5} & \multirow[t]{5}{*}{699} & \multirow[t]{5}{*}{52,48} \\
\hline & & R.2: 1 & 5 & & & \\
\hline & & R.3: 3 & 15 & & & \\
\hline & & R.4: 9 & 45 & & & \\
\hline & & R.5: 5 & 25 & & & \\
\hline \multirow[t]{5}{*}{ Étape de réalisation } & \multirow[t]{5}{*}{4} & R.1: 4 & 20 & \multirow[t]{5}{*}{4} & \multirow[t]{5}{*}{579} & \multirow[t]{5}{*}{43,46} \\
\hline & & R.2: 2 & 10 & & & \\
\hline & & R. $3: 2$ & 10 & & & \\
\hline & & R. $4: 3$ & 15 & & & \\
\hline & & R.5: 9 & 45 & & & \\
\hline \multirow[t]{5}{*}{ Étape d'intégration } & \multirow[t]{5}{*}{3} & R.1: 1 & 5 & \multirow[t]{5}{*}{3} & \multirow[t]{5}{*}{32} & \multirow[t]{5}{*}{2,40} \\
\hline & & R.2: 6 & 30 & & & \\
\hline & & R.3: 7 & 35 & & & \\
\hline & & R.4: 5 & 25 & & & \\
\hline & & R.5: 1 & 5 & & & \\
\hline \multirow[t]{5}{*}{ Après le cours } & \multirow[t]{5}{*}{1} & R.1: 5 & 25 & \multirow[t]{5}{*}{1} & \multirow[t]{5}{*}{0} & \multirow[t]{5}{*}{0,00} \\
\hline & & R.2: 8 & 40 & & & \\
\hline & & R.3: 5 & 25 & & & \\
\hline & & R.4: 0 & 0 & & & \\
\hline & & R.5: 2 & 10 & & & \\
\hline Total & ----- & ----- & ----- & ----- & 1332 & 100,00 \\
\hline
\end{tabular}

Notes: Rang $1:$ moins fréquent; rang 5 : plus fréquent. 
Lorsque cet ordre est comparé à celui découlant de la mise en ordre des fréquences effectives des $\mathrm{CP}$ (préparation: $\mathrm{N}=699$; réalisation: $\mathrm{N}=579$; intégration: $\mathrm{N}=32$; avant le cours : $\mathrm{N}=22$; après le cours: $N=0)$, la corrélation de rang de Spearman obtenue s'élève à $r_{s}=1,00(p<0,05)$ et s'avère significative. Ce résultat signifie que dans l'ensemble, la perception des stagiaires quant au moment où surviennent le plus fréquemment les $\mathrm{CP}$ est fiable et qu'elle semble représenter de manière fidèle la réalité décrite par le SOID.

\section{Discussion}

La gestion de l'indiscipline pose des défis particuliers en éducation physique et à la santé. En effet, cette matière scolaire se pratique dans des environnements diversifiés, bruyants, vastes et ouverts et se caractérise par la mise en œuvre d'actions motrices impliquant la manipulation d'objets, l'opposition directe et physique entre participants, des déplacements rapides et explosifs (Corriveau, Lirette et Laurencelle, 1991). En outre, il peut arriver que les composantes compétitives de certaines activités puissent exacerber des manifestations d'agressivité (Fernandez-Balboa, 1991). Ainsi, la gestion de l'indiscipline en éducation physique et à la santé recèle, en plus des enjeux rattachés à la couverture des programmes, au temps d'apprentissage et à l'apprentissage en lui-même, des enjeux reliés à la sécurité physique des élèves (Corriveau et al., 1991; Siedentop, 1994 ; Turcotte et Spallanzani, 2006).

Le caractère mouvant des situations d'enseignement-apprentissage dans cette matière ainsi que leur forte saturation en informations, surtout lors des moments éducatifs, des transitions et des jeux, sont des hypothèses plausibles que l'on peut avancer pour expliquer que l'accessibilité des $\mathrm{CP}$ allait en décroissant à mesure que leur intensité tendait à s'accroitre. En outre, il est prouvé que les $\mathrm{CP}$ se manifestent surtout de manière non verbale plutôt que verbale (Corriveau et al., 1991 ; Kennedy, 1982). Ceci accroit l'importance de l'observation active, du positionnement et des déplacements stratégiques (Desbiens, 2002) comme habiletés de supervision active permettant aux stagiaires d'avoir un meilleur accès aux CP susceptibles de survenir. Or, si les données disponibles dans la présente étude ne permettent pas de juger de ces trois habiletés, l'hypothèse qu'elles ne soient pas bien maitrisées ne peut non plus être écartée.

Rappelons, en effet, que les cours dirigés par les stagiaires étaient passablement perturbés puisque $51,66 \%$ des CP étaient de niveau 3, 29,20\% de niveau 2 et 19,14\% de niveau 1 . Les résultats, bien que non significatifs au plan statistique, suggèrent en outre que les stagiaires observés comme groupe ont une représentation imprécise du degré d'indiscipline qui prévaut durant leurs cours puisqu'ils ont indiqué à près de 55, 0 \% (54,54\%) qu'ils percevaient que les CPN1 étaient les plus nombreux alors que les CPN3 perçus les plus fréquents par $27,26 \%$ des stagiaires surviennent dans les faits presque trois fois plus souvent que les CPN1.

Une analyse plus fine examinant les relations entre la fréquence effective de chacun des $19 \mathrm{CP}$ et la perception de leur présence ou de leur absence durant le cours confirme l'imprécision de la représentation que les stagiaires se sont construites à propos du degré d'indiscipline. En effet, il ressort que les perceptions correspondent aux fréquences observées dans seulement $26,32 \%$ des cas (5/19 CP). Lorsque l'on examine de manière plus approfondie les cinq CP concernés, on constate que leur fréquence respective d'apparition est faible puisqu'elle varie entre $0,8 \%$ («Pas de costume ») et $6,8 \%$ («S'en prend au matériel $)$ ). Partant de ce qui précède, on peut faire l'hypothèse 
que le poids d'un CP dans l'économie globale de la gestion de l'indiscipline n'est pas uniquement lié à sa fréquence d'apparition. Il serait aussi relié à sa signification aux yeux du professionnel de l'éducation physique qui est responsable, par exemple, de signaler à la direction de l'établissement les élèves absents ou en retard $(\mathrm{N}=13$ ou 1,0\%), de contrôler les entrées et les sorties de son plateau ( $\mathrm{N}=14$ ou 1,1\%) ou encore de faire en sorte que les interactions entre les élèves de même qu'entre les élèves et lui correspondent aux normes du milieu scolaire («Est grossier»: N $=42$ ou 3,2\%). Ces éléments semblent rejoindre les observations de Kokkinos et al. (2004), selon qui les stagiaires seraient plus attentifs à certains $\mathrm{CP}$ qu'à d'autres. Ils confirment également la pertinence de dépasser la description statistique des phénomènes et de déployer parallèlement des dispositifs conduisant à explorer l'expérience subjective des situations des acteurs scolaires dont celle des enseignants et des stagiaires.

Les perceptions des stagiaires quant au moment où surviennent le plus fréquemment les $\mathrm{CP}$ sont, quant à elles, caractérisées par davantage de précision. En effet, ils ont été en mesure d'identifier les étapes de préparation et de réalisation comme étant celles où surviennent le plus de CP. Ces appréciations ont été formulées par au moins $70 \%$ des répondants (étape de préparation: $\mathrm{N}=13$ sur 20; étape de réalisation: $60 \%$ ou 12 sur 20), mais il faut relever que la variabilité d'un rang à l'autre pour un même comportement peut osciller entre $0 \%$ et $40 \%$. La corrélation de rang calculée entre la perception de la fréquence des $\mathrm{CP}$ et la fréquence effective des $\mathrm{CP}$ en fonction des moments du cours conduit dans ce cas à conclure que les perceptions des stagiaires quant aux moments où surviennent les $\mathrm{CP}$ indépendamment de leur degré d'intensité sont fiables et que leur capacité de se faire une idée viable de l'écologie de la séance à différents moments est bonne dans l'ensemble mais pour le moins variable. Nous n'avons pas de données permettant de se situer dans une perspective diachronique, mais il est possible que, comme l'a suggéré Belka (1988) en début d'article, les stagiaires de notre échantillon aient pu améliorer leurs capacités d'observation et d'interprétation des situations d'apprentissage à mesure qu'ils ont avancé dans leur programme de formation. Cet aspect mérite d'être approfondi.

\section{Conclusion}

Le stage est une de ces expériences qui marque l'individu autant par la répétition que par l'intensité de ce qui y est vécu. Nul doute que chaque épisode d'indiscipline des élèves est un des moments potentiellement marquants à l'intérieur de ces expériences culminantes que sont les stages car «rien n'est plus fatigant et contraignant pour un enseignant que d'avoir à faire face continuellement à des problèmes de discipline» (Siedentop, 1994, p. 142).

Contrairement à un mythe fréquemment véhiculé, le stagiaire ne peut pas à lui seul, indépendamment d'un encadrement approprié, parvenir à construire des savoirs d'action élaborés que ce soit en matière de gestion de l'indiscipline ou autres (Brunelle et Brunelle, 1999). Cet encadrement relève, nous semble-t-il, de deux aspects entretenant idéalement des liens d'interdépendance. Le premier concerne l'accompagnement individualisé dont le stagiaire peut bénéficier dans son lieu de stage de la part d'un enseignant associé. De par les interventions de ce dernier sur les conditions de réalisation du stage, les croyances et les perceptions du stagiaire, l'enseignant associé peut graduellement l'aider à développer sa capacité d'autoréguler son action en l'engageant dans des activités propices à l'auto-observation des conditions d'apprentissage offertes aux élèves, à l'autodiagnostic des raisons qui pourraient expliquer les résultats de l'observation et à l'autoformulation d'hypothèses d'action (Brunelle, Drouin, Godbout et Tousignant, 1988). 
Le deuxième aspect concerne l'accompagnement de groupe au moyen de séminaires de retour sur les stages visant l'exercice des capacités de réflexion critique des stagiaires. Ces séminaires peuvent prendre diverses formes, mais selon Turcotte et Spallanzani (2006, p. 19), la conduite d'activités d'auto-observation en différé à l'aide de la vidéoscopie mobilisant des cadres de référence signifiants comme le SOID et mettant à profit des interactions structurées entre stagiaires assurent «un raffinement des activités de formation vécues en gestion de classe en proposant une réflexion systématique sur l'intégration des savoirs, des savoir-faire et des savoir-être dans les situations professionnelles ».

Il ressort des travaux de Spallanzani et Tousignant (1990) que des activités de formation peuvent améliorer les capacités des étudiants en formation à l'enseignement de l'éducation physique et à la santé à mieux discriminer le degré de coopération des apprenants de même qu'à utiliser des concepts comme le temps d'engagement ou le taux d'attente des élèves. Les résultats de la présente étude montrent que, arrivés en fin de parcours de formation initiale, les stagiaires parviennent à se créer une représentation assez fiable de l'écologie des situations d'enseignement-apprentissage, mais que des améliorations peuvent encore être apportées aux modalités de formation de manière à ce que les stagiaires deviennent de meilleurs observateurs et interprètes des comportements des élèves.

\section{Références}

Belka, D.E. (1988). What preservice physical educators observe about lessons in progressive field experiences. Journal of Teaching in Physical Education, 7, 311-326.

Bell, R., Barrett, K.R. et Allison, P.C. (1985). What preservice physical education teachers see an unguided, early field experience. Journal of Teaching in Physical Education, 4, 81-90.

Broning, J.L. et Kintz, B.L. (1987). Computational handbook of statistics (3 éd.). Londres : Harper et Collins.

Brunelle, J et Brunelle J.-P. (1999). Un système de supervision de la maîtrise des compétences de base en enseignement de l'éducation physique et à la santé. Document de travail. Sherbrooke: Université de Sherbrooke, Faculté d'éducation physique et sportive.

Brunelle, J., Brunelle, J.-P., Gagnon, J., Goyette, R., Martel, D., Marzouk, A. et Spallanzani, C. (1993). Système d'incidents disciplinaires (SOID) ( $3^{\mathrm{e}}$ version). Document de présentation. Québec: Université Laval, Faculté des sciences de l'éducation, Département d'éducation physique, Groupe de recherche et d'intervention en activités physiques (GRIAP).

Brunelle, J., Drouin, D., Godbout, P. et Tousignant, M. (1988). La supervision de l'intervention en activité physique. Boucherville: Gaëtan Morin.

Chouinard, R. (1999). Enseignants débutants et pratiques de gestion de classe. Revue des sciences de l'éducation, $X X V(3)$, p. 497-514.

Copeland, W.D. (1987). Classroom management and student teachers' cognitive abilities: A relationship. American Educational Research Journal, 24(2), 219-236.

Corriveau, S., Lirette, M. et Laurencelle, L. (1991). Comportements perturbateurs des élèves dans les cours d'éducation physique au primaire. In R. Boileau (dir.), Éduquer comme profession... C'est quoi? Québec: Éditions l'Impulsion.

Desbiens, J.-F. (2002). L'évaluation et la responsabilisation des élèves : ébauche d'une problématique sur le thème de la supervision active en enseignement de l'éducation physique. Brock Education, 12(1), 36-48.

Desbiens, J.-F. et Spallanzani, C. (2005). La supervision active: un concept-clé pour mieux comprendre et agir sur l'écologie de la classe d'éducation physique et sportive. In C. Gervais et L. Portelance (dir.), Des savoirs au cour de la profession enseignante (p. 245-264). Sherbrooke: Éditions du CRP.

Doyle, W. (1986). Classroom organization and management. In M.C. Wittrock (dir.), Handbook of research on teaching (p. 392-431). New York, NY : Macmillan. 
Fernandez-Balboa, J.M. (1991). Beliefs, interactive thoughts, and actions of physical education students teachers regarding pupil misbehaviors. Journal of Teaching in Physical Education, 11(1), 59-78.

Fortier, A. et Desrosiers, P. (1991). Les préoccupations personnelles des stagiaires en éducation physique au primaire. STAPS, 26, 47-59.

Goyette, R., Doré, R. et Dion, É. (1997). Les stagiaires et la gestion des comportements perturbateurs. Cahiers de la recherche en éducation, 4(2), 223-297.

Graham, K.C., French, K.E. et Woods, A.M. (1993). Observing and interpreting teaching-learning processes: Novice PETE students, experienced PETE students and expert teacher educators. Journal of Teaching in Physical Education, 13, 46-61.

Kennedy, E.F. (1982). Discipline in the physical education setting. The Physical Educator, mai, 91-94.

Kokkinos, C.M., Panayiotou, G., Davazoglou, A.M. (2004). Perceived seriousness of pupils' undesirable behaviours: the student teachers' perspective. Educational Psychology, 24(1), 109-120.

Legault, F. (1999). La gestion de classe durant un stage d'initiation à l'enseignement et l'émergence d'une communauté virtuelle axée sur la résolution de problème. Revue des sciences de l'éducation, XXV(3), 593-619.

Martineau, S., Gauthier, C. et Desbiens, J.-F. (2000). Ce n'est pas toujours de la faute à El Nino. À propos de l'incompétence en enseignement. In C. Lessard et C. Gervais (dir.), L'évaluation des nouveaux programmes de formation des maîtres: une compétence à développer (p. 299-332). Montréal: Les publications de la Faculté des sciences de l'éducation de l'Université de Montréal.

(s.a.) (2004). Statistical package for social sciences (SPSS). Chicago, Il: Appache Software Foundation.

Siedentop, D. (1994). Apprendre à enseigner l'éducation physique (trad. par M. Tousignant, P. Boudreau et A. Fortier). Montréal: Gaëtan Morin.

Siegel, S. et Castellan, N.J. (1988). Nonparametric statistics for the behavioral sciences (2 éd.). Boston, MA: McGraw-Hill.

Spallanzani, C. et Tousignant, M. (1990). L'efficacité de l'enseignement telle que perçue par des éducateurs physiques en formation. Revue des sciences de l'éducation, XVI(3), 433-450.

Sprinthall, R.C. (2000). Basic statistical analysis (6 éd.). Boston, MA: Allyn and Bacon.

Stafford, J. et Bodson, P. (2006). L'analyse multivariée avec SPSS. Québec: Presses de l'Université du Québec.

Turcotte, S. et Spallanzani, C. (2006). Approfondir la réflexion sur les pratiques de gestion de classe : l'utilisation de la vidéoscopie par des étudiantes et étudiants en éducation physique et à la santé. Formation et profession, 13(1), dossier thématique, 17-21.

Turcotte, S. et Spallanzani, C. (2007). Structuration et mise en œuvre d'un dispositif pédagogique axé sur l'analyse de pratique assistée par vidéo. Actes du 24e congrès de l'AIPU. Vers un changement de culture en enseignement supérieur. Regards sur l'innovation, la collaboration et la valorisation (p. 336-338). Montréal : AIPU/Université de Montréal. 
Annexe 1

Système d'observation des incidents disciplinaires (SOID)

\begin{tabular}{|c|c|c|c|c|c|c|c|c|c|c|c|}
\hline \multicolumn{2}{|c|}{$\begin{array}{c}\text { Comportements perturbateurs } \\
\text { des élèves (1-2) }\end{array}$} & \multirow[t]{2}{*}{$\begin{array}{l}\text { Moments de la } \\
\text { leçon (3) }\end{array}$} & \multirow[t]{2}{*}{$\begin{array}{c}\text { Accessible ou } \\
\text { non accessible } \\
(4) \\
\end{array}$} & \multicolumn{3}{|c|}{$\begin{array}{l}\text { Effets compor- } \\
\text { tements } \\
\text { perturbateurs (5) }\end{array}$} & \multirow[t]{2}{*}{$\begin{array}{l}\text { Réactions du sta- } \\
\text { giaire (6) }\end{array}$} & \multicolumn{3}{|c|}{$\begin{array}{c}\text { Effets réactions } \\
\text { (7) }\end{array}$} & \multirow[t]{2}{*}{$\begin{array}{c}\text { Nombre } \\
\text { d'élèves }(8)\end{array}$} \\
\hline & & & & $\mathrm{I}$ & $\mathrm{NI}$ & $\mathrm{NC}$ & & $\mathrm{M}$ & NM & $\mathrm{NC}$ & \\
\hline 1. Est distrait & 13. Critique & 2. Introduction & $\begin{array}{l}\text { 2. Non accessi- } \\
\text { ble }\end{array}$ & & & & $\begin{array}{l}\text { 1. Dicte compor- } \\
\text { tement }\end{array}$ & & & & 2. 2 à 3 \\
\hline 4. Pas costume & $\begin{array}{l}\text { 16. Conduite dange- } \\
\text { reuse }\end{array}$ & 5. Transition & & & & & $\begin{array}{l}\text { Libertaire/ } \\
\text { Permissivité }\end{array}$ & & & & \\
\hline 5. Quitte salle & 17. Est grossier & 6. Éducatif & & & & & 4. Fait un rappel & & & & \\
\hline $2^{\mathrm{e}}$ niveau & 18. Ridiculise & 7. Jeu & & & & & 5. Ignore & & & & \\
\hline 8. Harcèle & & 10. Après le cours & & & & & 7. Donne raison & & & & \\
\hline 9. Fait du bruit & & & & & & & $\begin{array}{l}\text { Interactive/ } \\
\text { Ouverture }\end{array}$ & & & & \\
\hline 10. Déforme activité & & & & & & & $\begin{array}{l}\text { 8. Décrit } \\
\text { comportement }\end{array}$ & & & & \\
\hline $\begin{array}{l}\text { 11. Enfreint volon- } \\
\text { tairement règles }\end{array}$ & & & & & & & $\begin{array}{l}\text { 9. Exprime senti- } \\
\text { ments }\end{array}$ & & & & \\
\hline \multirow[t]{2}{*}{ 12. Arrête pratiquer } & & & & & & & $\begin{array}{l}\text { 10. Reconnait sen- } \\
\text { timents }\end{array}$ & & & & \\
\hline & & & & & & & $\begin{array}{l}\text { 11. Suscite arran- } \\
\text { gement }\end{array}$ & & & & \\
\hline
\end{tabular}


Annexe 2

Perception des catégories de moments d'apparition des comportements perturbateurs des élèves

Nom du stagiaire : École:

Voici cinq catégories de moments d'une leçon d'éducation physique au cours desquelles peuvent survenir des comportements perturbateurs. Quelles sont en ordre d'importance les catégories de moments de la leçon pendant lesquelles se sont manifestés les comportements perturbateurs des élèves du groupe observé pour le projet de recherche?

Indiquez en ordre croissant les cinq catégories de moments d'apparition des comportements perturbateurs, le rang 1 correspondant à la catégorie de moments présentant le moins de comportements perturbateurs et le rang 5 correspondant à la catégorie de moments présentant le plus de comportements perturbateurs.

\section{Catégorie 1}

\begin{tabular}{|c|c|c|}
\hline Avant le cours & $\begin{array}{l}\text { - Au vestiaire } \\
\text { - Sur le plateau avant le début de la séance }\end{array}$ & Rang: \\
\hline \multicolumn{3}{|l|}{ Catégorie 2} \\
\hline Préparation & $\begin{array}{l}\text { - Introduction } \\
\text { - Échauffement } \\
\text { - Explication } \\
\text { - Transition }\end{array}$ & Rang: \\
\hline \multicolumn{3}{|l|}{ Catégorie 3} \\
\hline Réalisation & $\begin{array}{l}\text { - Éducatif } \\
\text { - Jeu }\end{array}$ & Rang: \\
\hline \multicolumn{3}{|l|}{ Catégorie 4} \\
\hline Intégration & $\begin{array}{l}\text { - Retour au calme } \\
\text { - Conclusion }\end{array}$ & Rang: \\
\hline \multicolumn{3}{|l|}{ Catégorie 5} \\
\hline Après le cours & $\begin{array}{l}\text { - Au vestiaire } \\
\text { - Sur le plateau à la fin de la séance }\end{array}$ & Rang: \\
\hline
\end{tabular}




\section{Perception des comportements perturbateurs des élèves}

Nom du stagiaire: École :

Voici des comportements perturbateurs qui surviennent en éducation physique. Quels sont les comportements perturbateurs qui ont été adoptés par les élèves du groupe observé?

1) Précisez la présence de chacun des comportements perturbateurs lors de votre enseignement.

2) Indiquez l'ordre d'importance des trois niveaux de comportements perturbateurs en fonction de la fréquence d'apparition des comportements perturbateurs : le rang 1 correspond au degré d'intensité des comportements perturbateurs ayant été le moins fréquent et le rang 3 correspond au degré d'intensité des comportements perturbateurs ayant été le plus fréquent.

\begin{tabular}{|c|c|c|c|}
\hline \multirow[t]{2}{*}{ Comportements perturbateurs de $1^{\text {er }}$ niveau } & \multicolumn{2}{|c|}{ Présence } & \multirow[t]{2}{*}{ Rang } \\
\hline & Oui & Non & \\
\hline \multicolumn{4}{|l|}{ 1) Est distrait } \\
\hline \multicolumn{4}{|l|}{ 2) Bavarde } \\
\hline \multicolumn{4}{|l|}{ 3) Arrive en retard } \\
\hline \multicolumn{4}{|l|}{ 4) Pas de costume } \\
\hline \multicolumn{4}{|l|}{ 5) Quitte la salle } \\
\hline \multirow[t]{2}{*}{ Comportements perturbateurs de $2^{\mathrm{e}}$ niveau } & \multicolumn{2}{|c|}{ Présence } & Rang: \\
\hline & Oui & Non & \\
\hline \multicolumn{4}{|l|}{ 6) Fait le clown } \\
\hline \multicolumn{4}{|l|}{ 7) Se chamaille } \\
\hline \multicolumn{4}{|l|}{ 8) Harcèle } \\
\hline \multicolumn{4}{|l|}{ 9) Fait du bruit } \\
\hline \multicolumn{4}{|l|}{ 10) Déforme l'activité } \\
\hline \multicolumn{4}{|l|}{ 11) Enfreint volontairement les règles d'un jeu } \\
\hline \multicolumn{4}{|l|}{ 12) Arrête de pratiquer } \\
\hline \multirow[t]{2}{*}{ Comportements perturbateurs de $3^{\mathrm{e}}$ niveau } & \multicolumn{2}{|c|}{ Présence } & Rang: \\
\hline & Oui & Non & \\
\hline \multicolumn{4}{|l|}{ 13) Critique } \\
\hline \multicolumn{4}{|l|}{ 14) S'en prend au matériel } \\
\hline \multicolumn{4}{|l|}{ 15) Agresse } \\
\hline \multicolumn{4}{|l|}{ 16) Se conduit dangereusement } \\
\hline \multicolumn{4}{|l|}{ 17) Est grossier } \\
\hline \multicolumn{4}{|l|}{ 18) Ridiculise } \\
\hline 19) Résiste à une consigne & & & \\
\hline
\end{tabular}


